\title{
THE CHARACTERS OF THE BINARY MODULAR CONGRUENCE GROUP
}

\author{
BY PHILIP C. KUTZKO \\ Communicated by Olga Taussky Todd, November 30, 1972
}

1. Introduction. The determination of the characters of the groups $S L\left(2, Z / p^{n} Z\right)$ where $p$ is an odd prime is of interest for several reasons, among them the role that the group plays in the study of elliptic modular functions [1], [4], [5], [6]. Attempts have been made to compute the values of the irreducible characters of $S L\left(2, Z / p^{n} Z\right)$ and complete results were obtained by Shur [11] in the case $n=1$ and Praetorius [9] and Rohrbach [10] in the case $n=2$. Since that time analytic techniques involving theta-functions [7] and methods used for similar problems over locally compact groups [12] have been applied with partial results in the first case and a classification theorem in the second.

The purpose of the note is to announce that a complete description of the irreducible representations of $S L\left(2, Z / p^{n} Z\right)$ as well as the computation of the characters of these representations has been obtained by the author. These results comprise the author's Ph.D. Thesis (University of Wisconsin-1972) and will be published elsewhere.

2. Outline of results. Write $G_{n}$ for L.F. $\left(2, Z / p^{n} Z\right)$; i.e., let

$$
G_{n}=S L\left(2, Z / p^{n} Z\right) /\left\{ \pm\left(\begin{array}{ll}
1 & 0 \\
0 & 1
\end{array}\right)\right\} .
$$

For $r=1,2, \ldots, n$, let $K_{n, r}$ be the kernel of the homomorphism of $G_{n}$ onto $G_{r}$ that is the result of "reading" $G_{n}$ modulo $p^{r}$. Then $K_{n, r}$ is abelian of type $\left(p^{n-r}, p^{n-r}, p^{n-r}\right)$ whenever $r \geqq n / 2$. Now fix $n$ to be even and let $r=n / 2$. Write $e_{r}(x)$ for $\exp \left(2 \pi i x / p^{r}\right)$.

Definition 1 . Fix $l, 0 \leqq l \leqq p^{r}-1$ and write $l=p^{\alpha} l_{1}$ where $0 \leqq l_{1}$ $\leqq p^{r-\alpha}-1$ and $p \nmid l_{1}$ in case $l \neq 0$ and where we take $\alpha=r, l_{1}=1$ in case $l=0$. Write a typical element of $K_{n, r}$ in the form $I+p^{r}\left(\begin{array}{cc}a \\ c & -a \\ c\end{array}\right)$ where $I=\left(\begin{array}{ll}1 & 0 \\ 0 & 1\end{array}\right)$. Then $\psi_{l}$ is defined to be the character on $K_{n, r}$ which maps $I+p^{r(a}\left(\begin{array}{ll}b & -b\end{array}\right)$ to $e_{r}\left((l b+c) / 2 l_{1}\right)$.

THEOREM 1. $\psi_{l}$ may be extended to a character $\eta_{l}$ defined on the normalizer of $\psi_{l}$ (see [4] for definitions). The character $\times_{l}$ induced by $\eta_{l}$ on $G_{n}$ is irreducible. $\times_{1}$ does not contain $K_{n, n-1}$ in its kernel.

Having proved Theorem 1 it is a simple matter to determine con-

AMS (MOS) subject classifications (1970). Primary 20G05, 20H05, 15A33; Secondary $10 \mathrm{D} 05$.

Copyright (C) American Mathematical Society 1973 
ditions on $l$ such that the resulting set of characters $\times_{l}$ form a complete set of irreducible characters on $G_{n}$ which are not characters on $G_{n-1}$ and these characters may be computed with relative ease.

Now let $n>1$ be odd and write $r=(n+1) / 2$. As in the even case write a typical element of $K_{n, r}$ in the form $I+p^{r}\left(\begin{array}{cc}a & -a \\ c & -a\end{array}\right)$ and define $\psi_{l}$ on $K_{n, r}$ by $\psi_{l}\left(I+p^{r}\left(\begin{array}{cc}a & -b \\ c & -a\end{array}\right)\right)=e_{r-1}\left((l b+c) / 2 l_{1}\right)$. Here, $l=0,1, \ldots, p^{r-1}-1$. Write $T_{l}$ for the normalizer of $\psi_{l}$ in $G_{n}$. Then $\psi_{l}$ cannot be extended to $T_{l}$ and we must modify our techniques.

THEOREM 2. Let $p \nmid l$. Let $H_{l}$ be the subgroup of elements of $G_{n}$ of the form $\left(\begin{array}{c}t \\ l u\end{array}\right)$ and let $T_{l, 0}=H_{l} K_{n, r}$. Then $\psi_{l}$ can be extended to a character $\theta_{l}$ on $T_{l, 0}$. Let $\theta_{l}^{\prime}$ be the restriction of $\theta_{l}$ to $T_{l, 0}$ and let $X_{l}$ and $\times_{l}^{\prime}$ be the characters induced on $G_{n}$ by $\theta_{l}$ and $\theta_{l}^{\prime}$ respectively. Then $(l / p)\left(\times_{l}-2 / p \times_{l}^{\prime}\right)$ is an irreducible character on $G_{n}$ which does not contain $K_{n, n-1}$ in its kernel. Here, $(l / p)$ is the Legendre symbol.

We note that this construction is not as unnatural as it seems since in fact $T_{l}=H_{l} K_{n, r-1}$ and since the character constructed in Theorem 2 has the same character table as the character constructed in Theorem 1 in case $p \nmid l$.

THEOREM 3. Let $p \mid l$. Let $\bar{N}_{l}$ be the subset of elements of $G_{r}$ of the form $\left(\begin{array}{c}t \\ i \\ i\end{array}\right)\left(I+p^{r-1}\left(\begin{array}{cc}a & 0 \\ 0 & -a\end{array}\right)\right)$. Then $\bar{N}_{l}$ is in fact a subgroup of $G_{r}$. Let $N_{l}$ be the total inverse image of $\bar{N}_{l}$ in $G_{n}$. Then $\psi_{l}$ can be extended to a character $\theta_{l}$ on $N_{l}$ and the character induced by $\theta_{l}$ on $G_{n}$ is irreducible and does not contain $K_{n, n-1}$ in its kernel.

Again it is relatively simple to select a subset of the characters constructed in Theorems 2 and 3 which is precisely the set of irreducible characters on $G_{n}$ which are not characters of $G_{n-1}$.

Since the characters of $G_{1}$ are known [5], and since for any character $\times$ of $G_{n}$ ( $n$ even or odd) there is a $k$ such that $\times$ may be viewed as a character on $G_{k}$ but not on $G_{k-1}$. Theorems 1-3 determine all irreducible characters on $G_{n}$.

Finally, the methods outlined above may be utilized with minor modifications to determine the characters of $S L\left(2, Z / p^{n} Z\right)$ and one notes [8] that this suffices to determine the characters of $S L(2, Z / m Z)$ for any integer $m$.

\section{REFERENCES}

1. C. Chevalley and A. Weil, Über das Verhalten der Integrale erster Gattung bei Automorphismem des Funktionenkörpers, Abh. Math. Sem. Univ. Hamburg 10 (1934), 358-361.

2. W. Feit, Characters of finite groups, W. A. Benjamin, Inc., New York, 1967.

3. G. Frobenius, Uber Gruppencharaktere, S. -B. Berlin. Math. Akad. (1896), 985-1021.

4. R. C. Gunning, Lectures on modular forms, Ann. of Math. Studies, no. 48, Princeton, N.J. (1962). MR 24 \# A2664. 
5. E. Hecke, Uber ein Fundamental problem aus der Theorie der Elliptischen Modulfunktionen, Collected Works (1958), 525-547.

6. - Uber das Verhalten der Integrale erster Gattung bei Abbildungen, ibid., 548-558.

7. H. D. Kloosterman, The behaviour of general theta functions under the modular group and the characters of binary modular congruence groups. I and II, Ann. of Math. (2) 47 (1946), 317-447. MR 9, 12; 8, 10.

8. D. McQuillan, A generalization of a theorem of Hecke, Amer. J. Math. 84 (1962), 306-316. MR 25 \# 5041.

9. H. W. Praetorius, Die Charaktere der Modulargruppen der Stufe $q^{2}$, Abh. Math. Sem. Univ. Hamburg 9 (1933), 365-394.

10. H. Rohrbach, Die Charaktere der binaren Kongruenzgruppen mod $p^{2}$, Schr. Math. Sem. Inst. Math. Univ. Berlin. 1 (1932/33), 33-94.

11. J. Schur, Untersuchungen uber die Darstellung der endlichen Gruppen durch gebrochene lineare substitutionen, J. Reine Angew. Math. 132 (1907), 85-137.

12. S. Tanaka, Irreducible representations of the binary modular congruence groups mod p, J. Math. Kyoto Univ. 7 (1967), 123-132. MR 37 \#5311.

Department of Mathematics, Princeton University, Princeton, New Jersey 08540 